\title{
Equivalence between free quantum particles and those in harmonic potentials and its application to instantaneous changes
}

\author{
Ole Steuernagel ${ }^{\mathrm{a}}$ \\ School of Physics, Astronomy and Mathematics, University of Hertfordshire, Hatfield, AL10 9AB, UK
}

Received: 10 April 2014

Published online: 11 June 2014

(C) The Author(s) 2014. This article is published with open access at Springerlink.com

\begin{abstract}
In quantum physics the free particle and the harmonically trapped particle are arguably the most important systems a physicist needs to know about. It is little known that, mathematically, they are one and the same. This knowledge helps us to understand either from the viewpoint of the other. Here we show that all general time-dependent solutions of the free-particle Schrödinger equation can be mapped to solutions of the Schrödinger equation for harmonic potentials, both the trapping oscillator and the inverted "oscillator". This map is fully invertible and therefore induces an isomorphism between both types of system, they are equivalent. A composition of the map and its inverse allows us to map from one harmonic oscillator to another with a different spring constant and different center position. The map is independent of the state of the system, consisting only of a coordinate transformation and multiplication by a form factor, and can be chosen such that the state is identical in both systems at one point in time. This transition point in time can be chosen freely, the wave function of the particle evolving in time in one system before the transition point can therefore be linked up smoothly with the wave function for the other system and its future evolution after the transition point. Such a cut-and-paste procedure allows us to describe the instantaneous changes of the environment a particle finds itself in. Transitions from free to trapped systems, between harmonic traps of different spring constants or center positions, or, from harmonic binding to repulsive harmonic potentials are straightforwardly modelled. This includes some time-dependent harmonic potentials. The mappings introduced here are computationally more efficient than either state-projection or harmonic oscillator propagator techniques conventionally employed when describing instantaneous (non-adiabatic) changes of a quantum particle's environment.
\end{abstract}

\section{Introduction and motivation}

A quantum particle confined by a harmonic potential features a discrete energy spectrum; a free particle's energy can have any positive value. At first, it might sound implausible that these two cases are fully equivalent, yet, simple mathematics accessible at the undergraduate level suffices to prove this fact.

This equivalence provides a formal connection between the free and the trapped case helping us to understand features of one system from the point of view of the other [1], or why they share features [2]. It can also help with calculations. If analytical solutions for a trapped system are needed one can switch to the free particle picture since the propagators are easier to integrate and therefore closed form solutions may be determined which are otherwise inaccessible [3]; then one can map back to the trapped system. For numerical calculations the trapped particle picture can offer advantages because the confinement of the particle allows us to circumvent grid adaptation, often necessary for modelling of free particles spreading without bound. Here, particular attention is paid to the application of the equivalence for the modelling of instantaneous transitions. Three types of transitions can easily be described: instantaneously setting a particle free or capturing a free particle, instantaneous change of the spring constant or stiffness of a harmonic trap, including instantaneous switch-over to an inverted "oscillator", and instantaneous displacements of a trap.

That the cases of free and harmonically trapped quantum particle are connected seems to have been observed first in the field of optics, rather than in the field of mathematical physics or quantum mechanics. For instance Yariv's textbook [4] from 1967 introduces the well known freely propagating modes of laser beams and points out that they are

\footnotetext{
${ }^{a}$ e-mail: 0.Steuernagel@herts.ac.uk
} 
"of the same form" as the eigenstates of the harmonic oscillator. In mathematical physics their full equivalence appears to have been established first by Niederer in 1972 [5] using group theoretical arguments and by Takagi in 1990 [6] using coordinate transformations. Takagi's work additionally emphasises that a continuous change of spring constant of the harmonic binding potential over time can be modelled. An associated constant of motion for time-varying harmonic potentials was investigated by Lewis in 1967 [7]. In theoretical optics the equivalence between free and harmonically trapped particles was investigated by Nienhuis et al. in 1993 using operator methods [8]. The connection with the Gouy-phase of optics, the phase shift of $\pi$ (or multiples thereof) a beams suffers when going through a focus, was made explicit by Steuernagel in 2005 [1]. Barton's comprehensive 1986 article on the inverted "oscillator" [9] does not mention the equivalence although it also applies to such a system (which disperses a particle with a negative spring constant, i.e., a repulsive Hookean force). A connection to the inverted oscillator has been made in 2006 by Yuce and others [10]. Recently, interesting features of freely moving waves have been analyzed using the equivalence [11].

Here, we give one possible classical mechanics motivation for the equivalence in sect. 2. The map for instantaneous harmonic trapping of a free particle and its inverse is presented in sect. 3 , followed by its application to the modelling of instantaneous changes in the environment in sect. 4. It is one of the main motivations for this article to show that the equivalence can be used to describe instantaneous (non-adiabatic) changes in simple terms; it seems that this application has not been reported before although it is interesting [12,13]. A composition of different maps to model mappings between harmonic potentials of different spring constants is introduced in sect. 5 . This is followed by mappings between laterally shifted traps of different spring constants in sect. 6. Mappings to inverted "oscillators" in sect. 7 are followed by mappings to freely falling (uniformly accelerated) particles in sect. 8 . Section 9 shows that the approach described here cannot be extended to other systems such as particles in anharmonic potentials; the following sect. 10 does establish the class of time-dependent harmonic potentials free particles can be mapped to. We conclude with a summary of the usefulness of our results in sect. 11.

\section{Classical case}

To motivate the quantum case let us briefly consider the 1-dimensional classical harmonic oscillator with coordinate $\xi$, described by the Hamiltonian

$$
H=\frac{p^{2}}{2 M}+\frac{k}{2} \xi^{2}=\frac{1}{2 M}\left(p^{2}+M^{2} \omega^{2} \xi^{2}\right)
$$

where $M$ is the mass of the particle, $k$ the spring constant and $\omega=\sqrt{k / M}$ its resonance frequency. In suitable coordinates its phase space trajectories are circles, this suggests the coordinate transformation (paraphrasing Goldstein [14])

$$
\xi(X, P)=\sqrt{\frac{2 P}{M \omega}} \cos (X)
$$

and

$$
p(X, P)=-\sqrt{2 P M \omega} \sin (X) .
$$

These are known to form "canonical transformations", which implies that they map $H$ to the equivalent Hamiltonian $[14]$

$$
H^{\prime}=\omega P\left(\sin (X)^{2}+\cos (X)^{2}\right)=\omega P,
$$

which is independent of the coordinate $X$, that is, the generalized momentum $P$ is conserved. Using the Hamiltonian equation of motion $\frac{\mathrm{d}}{\mathrm{d} t} X=\partial H^{\prime} / \partial P=\omega$ yields

$$
X(t)=\omega t+\phi_{0}
$$

Upon insertion into eq. (3), eq. (5) not only yields the correct solution but structurally it resembles the motion of a free particle $x(t)=v t+x_{0}$.

To extract time via the phase angle of a harmonic oscillator one can form the arctangent of its circular motion, it is therefore perhaps not surprising that the correct guess for the coordinate mapping for time in the quantum case involves tangent and arctangent functions, compare eqs. (7) and (12) below.

\section{Map from free to trapped case}

The Schrödinger equation for a free particle of mass $M$ in one spatial dimension $x$, described by a wave function $\phi(x, t)$ that depends on time $t$, is given by

$$
\left[-\frac{\hbar^{2}}{2 M} \frac{\partial^{2}}{\partial x^{2}}-i \hbar \frac{\partial}{\partial t}\right] \phi(x, t)=0,
$$

where $h$ is Planck's constant in $\hbar=h /(2 \pi)$. 
Since the wave functions for free particles and those subjected to harmonic potentials factorize with respect to their spatial coordinates, we will only discuss the one-dimensional case, rather than two or three dimensions.

The coordinate transformations $[6,1]$ from free to trapped system

$$
x(\xi, \tau)=\frac{\xi \sqrt{b \omega}}{\cos (\omega \tau)} \quad \text { and } \quad t(\tau)=b \tan (\omega \tau)
$$

conform with classical expectations, compare sect. 2. Here $b$ is a free parameter to be fixed below for the case of the modelling of instantaneous environmental changes. Applied to the mapping of solutions $\phi(x, t)$ of eq. (6), eqs. (7) yield the wave function of a harmonically trapped particle

$$
\psi(\xi, \tau)=\frac{\phi(x(\xi, \tau), t(\tau))}{f(x(\xi, \tau), t(\tau) ; b)},
$$

which contains the form factor

$$
f(x, t ; b)=\frac{\exp \left(\frac{i M t x^{2}}{2 \hbar\left(t^{2}+b^{2}\right)}\right)}{\left(1+t^{2} / b^{2}\right)^{1 / 4}},
$$

and solves Schrödinger's equation of a harmonic oscillator with spring constant $k$

$$
\left[-\frac{\hbar^{2}}{2 M} \frac{\partial^{2}}{\partial \xi^{2}}-i \hbar \frac{\partial}{\partial \tau}+\frac{k}{2} \xi^{2}\right] \psi(\xi, \tau)=0
$$

This is straightforward (and tedious) to check by direct substitution.

Transformation (7) maps $t \in(-\infty, \infty)$ onto $\tau \in(-\pi / 2, \pi / 2)$ only, but the periodicity arising through the use of trigonometric functions represents the oscillator's motion for all times $\tau$.

It is noteworthy that the discontinuities of the transformation (7) map from a Schrödinger equation with a continuous to another with a discrete energy spectrum. Time and energy being canonical conjugates, this conspires to give us the discrete energy spectrum of the harmonic oscillator.

We note that the term $\left(1+t^{2} / b^{2}\right)^{-1 / 4}$ occurs in the form factor (9) to preserve the normalization of the mapped wave function. Its numerator can be correctly guessed when one attempts to cancel terms containing the factor $\xi \frac{\partial \phi}{\partial \xi}$. Otherwise such terms arise in eq. (10); compare the discussion following eq. (37) in sect. 9 below.

\section{Inverse map (trapped to free)}

The coordinate transformations inverse to (7) are

$$
\xi=\frac{x}{\sqrt{b \omega} \sqrt{\left(1+t^{2} / b^{2}\right)}}
$$

and

$$
\tau=\frac{1}{\omega} \arctan \left(\frac{t}{b}\right),
$$

and go together with the wave function multiplication $\phi=\psi f$, inverting eq. (8) and thus mapping from eq. (10) to eq. (6).

\section{Instantaneous changes of the environment}

If one wants to model instantaneous changes happening to the environment of a quantum particle one generally has to decompose the wave functions of the system at the time of the transition into the superposition of eigenfunctions of the "new" system and then determine their time evolution in the new system. This can involve sums over infinitely many eigenstates of the new system making exact results hard to obtain, often forcing us to truncate expressions.

In contrast, for maps between systems with potentials up to second order in $\xi$, the approach discussed here can model instantaneous transitions of the potential with ease. For several successive instantaneous changes one ends up with the following sequence: determine the initial state, then its time evolution in the free case, map onto the trapped case (if that is to be modelled), determine its final state at the next transition point in time $t_{0}$, this serves as the initial state of the next free propagation-step, etc. 


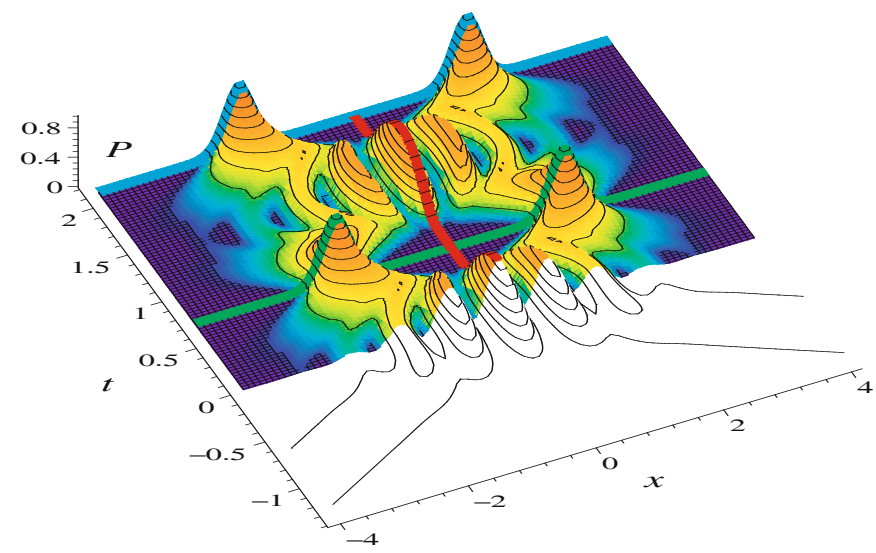

Fig. 1. Probability density of a free quantum particle in an equal superposition of two Gaussian states $P(x, t) \sim \mid \phi_{0}\left(x, t ; 0, p_{0}\right)+$ $\left.\phi_{0}\left(x, t ; 0,-p_{0}\right)\right|^{2}$ as described by eq. (16), with $\hbar=1, M=1, \sigma_{0}=3 / 2$, and opposing momenta $p_{0}=4$. At time $t=\tau=0$ the particle instantaneously gets trapped by a potential with spring constant $k=5$. The motion of the two half waves leads to interference near the origin of the potential (centered on red line at $x_{0}=\xi_{0}=0$ ). This plot illustrates that the trapped particle ends up in a superposition of two squeezed coherent states [15]. The squeezing (narrowing of peaks) is evident at times $T / 4$ and $3 T / 4$ after the transition time (green and blue cross lines; $T=2 \pi / \sqrt{5} \approx 2.81$ ).

It is desirable to shift the origin of time to each respective transition point $t_{0}=0$ and it is necessary to choose the coordinate stretching factor $b$ in such a way that the wave function is momentarily unchanged at the transition point. Without loss of generality the specific choices

$$
\tau_{0}=\tau\left(t_{0}\right)=\tau(0)=0
$$

and

$$
b=1 / \omega=\sqrt{M / k}
$$

yield such a smooth mapping since, according to eq. (7), in this case

$$
x\left(t_{0}\right)=\xi\left(\tau_{0}\right) .
$$

Equation (15) assures that the two branches, say $\phi(x, t)$ for $t<t_{0}=0$ and $\psi(\xi, \tau)$ for $\tau>\tau_{0}=0$, can be glued together continuously $\phi(x, 0)=\psi(\xi, 0)$, since additionally for the "smooth" choice $b=1 / \omega$ the form factor $f\left(x, t_{0} ; \frac{1}{\omega}\right)=1$.

Note that the mapping implies that the entire environment of the particle changes instantaneously, irrespective of the particle's quantum state. The energy of the particle is not conserved: mapping from eq. (6) to eq. (10) increases the particle's total energy by the potential energy $\langle V\rangle=\int \mathrm{d} x \overline{\psi\left(\xi, t_{0}\right)} \frac{k}{2} \xi^{2} \psi\left(\xi, t_{0}\right)$ (the overbar denotes complex conjugation), the reverse map correspondingly reduces it. This is quite different to the textbook scenario where a particle approaches a region in space where the potential, at some location $x_{0}$, steps abruptly to a different potential. In that case the particle's energy remains unchanged but its wave function changes abruptly, including being reflected at the step.

To illustrate the application of the mapping to a instantaneous change of the environment we use the well-known textbook example of a freely evolving Gaussian wave packet with initial position spread $\sigma_{0}$

$$
\phi_{0}\left(x, t ; x_{0}, p_{0}\right)=\frac{1}{\sqrt{\sqrt{\pi} \sigma(t)}} \exp \left[-i M v_{0} \frac{\left(x-x_{0}\right) \sigma_{0}}{\hbar \sigma(t)}\right] \times \exp \left[-\frac{\left(x-x_{0}\right)^{2}}{2 \sigma_{0} \sigma(t)}-i \frac{M v_{0}^{2}}{2} \frac{t \sigma_{0}}{\hbar \sigma(t)}\right] \text {, }
$$

where

$$
\sigma(t)=\sigma_{0}+i \frac{t \hbar}{\sigma_{0} M}
$$

Here $x_{0}$ parameterizes spatial and $p_{0}=M v_{0}$ momentum displacement of the wave functions. If either of these two quantities is non-zero the mapping onto a harmonically trapped state results in a state with oscillating center-of-mass. In general, although it is a wave function of Gaussian shape, i.e. a coherent state, this freely evolving wave packet will not "fit" the width of the harmonic potential and therefore be "squeezed". In short, the state of eq. (16) trapped in a harmonic potential becomes a squeezed coherent state [15], see cross lines in fig. 1. 


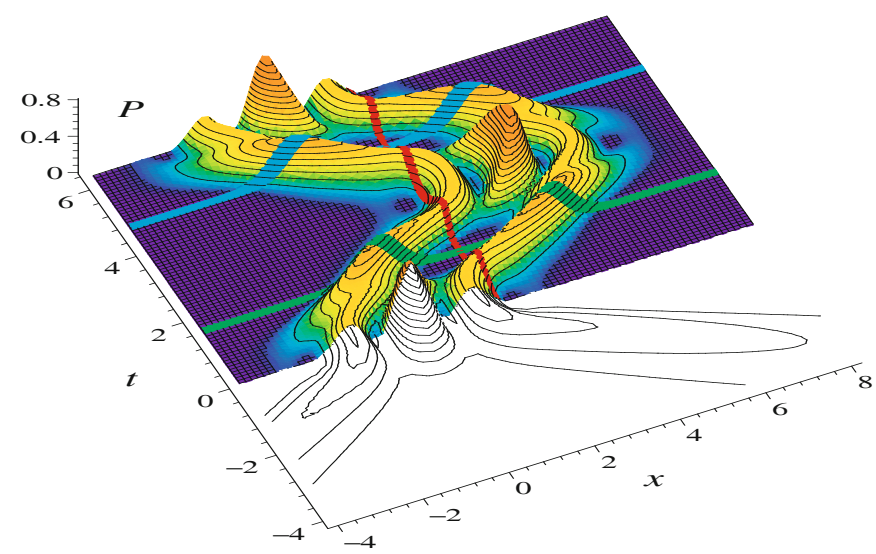

Fig. 2. Same as fig. 1 above with the center of the trapping potential, indicated by the solid red line, laterally shifted to $\xi_{0}=2$. Here $p_{0}=2$ and $k=1(T=2 \pi \approx 6.28)$.

\section{Composed maps (trapped to trapped)}

The composed coordinate transformations from an initial harmonic trapping potential with spring constant $k$ and wave function $\psi(\xi, \tau ; k)$, via the free particle-case $\phi$, to a final harmonic potential with spring constant $K$ and wave function $\Psi(\xi, \tau ; K)$ is given by

$$
\begin{aligned}
\Xi & =\frac{\xi}{\sqrt{\cos ^{2}(\Omega \tau)+\frac{k}{K} \sin ^{2}(\Omega \tau)}}, \\
T & =\frac{1}{\omega} \arctan \left(\sqrt{\frac{k}{K}} \tan (\Omega \tau)\right),
\end{aligned}
$$

and

$$
\Psi(\xi, \tau ; K)=\psi(\Xi(\xi, \tau), T(\tau) ; k) \times \frac{f\left(x(\xi, \tau), t(\tau) ; \frac{1}{\omega}\right)}{f\left(x(\xi, \tau), t(\tau) ; \frac{1}{\Omega}\right)} .
$$

Here $\Omega=\sqrt{K / M}$, and $\Psi(\xi, \tau ; K)$ solves the Schrödinger equation (10) with $k$ substituted by $K$.

It can be checked that the inverse of the coordinate transformations (18) and (19) are given by the same functional expressions with the quantities pertaining to one potential swapped with those of the other $(\psi \leftrightarrow \Psi, k \leftrightarrow K$ and $\omega \leftrightarrow \Omega)$

\section{Laterally shifted traps}

The coordinate transformation (7) can also incorporate a lateral shift of the trapping potential. Direct substitution shows that the mapping

$$
x\left(\xi-\xi_{0}, \tau\right)=\frac{\left(\xi-\xi_{0}\right)}{\cos (\tau \omega)}
$$

applied to eqs. (8) and (9) yields Schrödinger's eq. (10) for the harmonic oscillator with a shifted trapping potential: $k \xi^{2} \mapsto k\left(\xi-\xi_{0}\right)^{2}$. This observation allows us to model instantaneous transition of a particle from the free state or a trap centered at some initial position to another trap with different stiffness and center position, compare fig. 1 with fig. 2. In order to implement this map we use eq. (21) to map the position shift over into the new set of coordinates of the displaced trapping potential and subsequently "undo" this shift by substracting $\xi_{0}$ from the argument of the wave function in order to make sure that outgoing and incoming waves match up at the transition time; compare discussion following eq. (14). Note that this latter compensation only applies to the wave function that is to be mapped, not the dividing factor $f\left(x, t ; \frac{1}{\omega}\right)$ : eq. (8) thus becomes

$$
\psi(\xi, \tau)=\frac{\phi\left(x\left(\xi-\xi_{0}, \tau\right)+\xi_{0}, t(\tau)\right)}{f\left(x\left(\xi-\xi_{0}, \tau\right), t(\tau) ; \frac{1}{\omega}\right)} .
$$

The same applies to eqs. (18) and (20). 


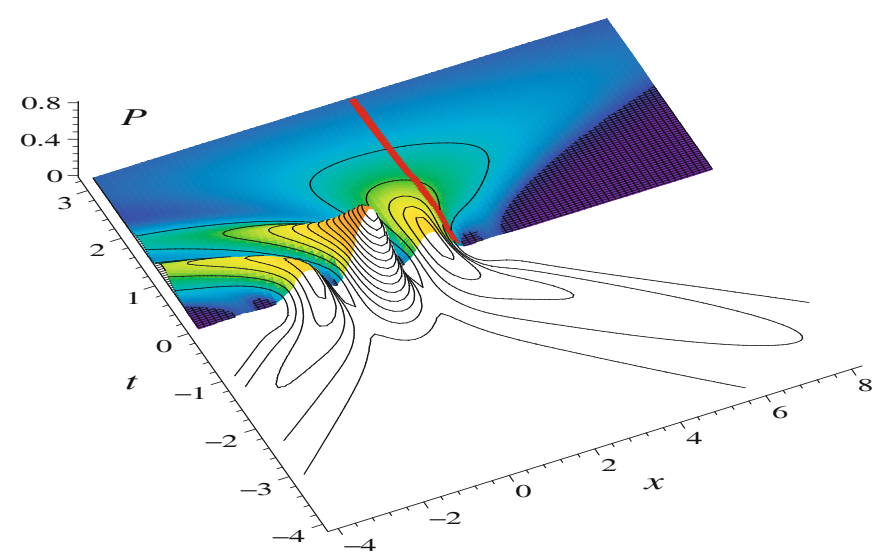

Fig. 3. Same as fig. 2 above except for the potential being inverted: $k=-1$.

\section{Inverted "oscillators"}

In his foundational work on inverted "oscillators" Barton [9] used that a regular harmonic oscillator can be converted into an inverted oscillator using the formal "complexification" $\omega \mapsto i \omega$. It was later realized by Yuce et al. [10] that a coordinate transformation like (7) allows for a map from free system to inverted harmonic potentials. Using Barton's substitution and the smooth choice $\left(b=\frac{1}{\omega}\right)$ eqs. (7) become

$$
x(\xi, \tau)=\frac{\xi}{\cosh (\tau \omega)} \quad \text { and } \quad t(\tau)=\frac{\tanh (\tau \omega)}{|\omega|} .
$$

Wave function map (8) and form factor (9) remain unchanged. This yields the harmonic oscillator Schrödinger equation (10) with spring constant $k \mapsto-k$ and can include the lateral shift (21) as well.

This case is illustrated in fig. 3 .

\section{From free to freely falling particle}

In order to provide further context for the transformations introduced here, we now consider the mapping from a free to a freely falling quantum particle. This simple case is readily treated with the approach advertised here. As before, the free particle with wave function $\phi(x, t)$ fulfils eq. (6), we substitute

$$
x(\xi, \tau)=\xi+\frac{a \tau^{2}}{2} \quad \text { and } \quad t(\tau)=\tau .
$$

Applied to the mapping of solutions of eq. (6)

$$
\psi(\xi, \tau)=\frac{\phi(x(\xi, \tau), t(\tau))}{g(x(\xi, \tau), t(\tau) ; a)},
$$

with

$$
g(x, t ; a)=\exp \left(i \frac{M a t}{\hbar}\left(x+\frac{a}{2} t^{2}\right)+i \frac{M a^{2}}{6 \hbar} t^{3}\right),
$$

this yields solutions $\psi(\xi, \tau)$ for the Schrödinger equation

$$
\left[-\frac{\hbar^{2}}{2 M} \frac{\partial^{2}}{\partial \xi^{2}}-i \hbar \frac{\partial}{\partial \tau}+M a \xi\right] \psi(\xi, \tau)=0,
$$

where $a$ is the acceleration of the freely falling particle.

\section{Can this method be generalized?}

Our investigations raise the question whether the method described here can be generalized to other potentials, perhaps to anharmonic potentials such as the solvable cases discussed in refs. [16] and [17]? 
One might think recent work by Costa Filho et al. [18] provides an example of just such a mapping from the harmonic case to that of the (anharmonic) Morse potential. But it turns out that the coordinate transformation employed there does not preserve the standard commutation relations of quantum physics (or Heisenberg's uncertainty principle [18]) rendering the transformation unphysical.

The phase space time evolution of a classical harmonic oscillator is known to amount to a rigid rotation because the oscillation frequency is independent of the state, the amplitude, of the oscillator. For anharmonic potentials this state independence does not apply which is why they are very different to harmonic potentials. Recent investigations of Wigner flow in quantum phase space [2] have established that anharmonic potentials show qualitatively very different quantum phase space flow behaviour from the rigid rotation that is found in the harmonic case. It therefore appears unlikely that a simple coordinate transformation could map from one to the other thus extending the equivalence to anharmonic potentials.

We will now show that, indeed, no mapping from the studied three equivalent cases (free particle, freely falling particle and particle in harmonic potential) to cases with anharmonic potentials exists. We first investigate the constraints on coordinate transformations of the general form

$$
x(\xi, \tau)=X(\xi, \tau) \quad \text { and } \quad t(\xi, \tau)=T(\xi, \tau) .
$$

The transformations equations (7), (12) and (24) introduced so far have in common that the mapped time $\tau$ only depends on the original time $T(\xi, \tau)=T(\tau)$. If $T$ depended on $\xi$ then $\partial T / \partial \xi \neq 0$ which in turn would imply that the differential operator $\partial^{2} / \partial x^{2}$, upon transformation to the new coordinates, "spills over" into the mapped time giving rise to a term $\partial^{2} / \partial \tau^{2}$ which cannot be cancelled by other terms. This can be shown by applying the chain rule or studying the Hessian of the transformation. Since second order time derivatives have no place in Schrödinger's equation we have established that the transformation for time never depends on the spatial coordinate.

With $X^{\prime}=\partial X(\xi, \tau) / \partial \xi, X^{\prime \prime}=\partial^{2} X(\xi, \tau) / \partial \xi^{2}$ and $\dot{T}=\partial T(\tau) / \partial \tau$, application of the chain rule yields

$$
\frac{\partial}{\partial t}=\frac{1}{\dot{T}} \frac{\partial}{\partial \tau}
$$

and

$$
\frac{\partial^{2}}{\partial x^{2}}=\frac{1}{X^{\prime 2}} \frac{\partial^{2}}{\partial \xi^{2}}-\frac{X^{\prime \prime}}{X^{\prime 3}} \frac{\partial}{\partial \xi}+O\left(\frac{\partial}{\partial \tau}\right),
$$

where $O\left(\frac{\partial}{\partial \tau}\right)$ refers to terms containing up to first order derivatives in $\tau$. Obviously, the transformation of partial derivatives "incurs" coefficient functions $C(\xi, \tau)$. The first coefficients in eqs. (29) and (30) need to be equal, that is

$$
C(\xi, \tau)=\frac{1}{\dot{T}}=\frac{1}{X^{\prime 2}}
$$

Only this allows us to extract the common factor $C$ guaranteeing that the mapping preserves the structure of Schrödinger's equation for the sum of total and kinetic energy

$$
-\frac{\hbar^{2}}{2 M} \frac{\partial^{2}}{\partial x^{2}}-i \hbar \frac{\partial}{\partial t} \mapsto C\left[-\frac{\hbar^{2}}{2 M} \frac{\partial^{2}}{\partial \xi^{2}}-i \hbar \frac{\partial}{\partial \tau}\right]+\mathcal{V}
$$

here $\mathcal{V}$ refers to all other terms. With eq. (31) we have established that

$$
T=\int \mathrm{d} \tau X^{\prime}(\xi, \tau)^{2}
$$

at the same time we know that $T$ must not depend on the spatial coordinate $\xi$, therefore $X$ is at most linear in $\xi$

$$
X(\xi, \tau)=A(\tau) \xi+B(\tau)
$$

and thus

$$
T(\tau)=\int_{\tau_{0}}^{\tau} \mathrm{d} \tilde{\tau} A(\tilde{\tau})^{2} .
$$

Here $A$ and $B$ are, at this stage, arbitrary functions of $\tau$ such that all transformations are invertible. We write the form factor $f$ as

$$
f(\xi, \tau)=\frac{\exp [i \epsilon(x(\xi, \tau), t(\xi, \tau))]}{\sqrt{N(\tau)}},
$$


where the normalization $N(\tau)=A(\tau)$ takes account of the stretching of the coordinate system and $\epsilon$ is real. This, respectively, enforces normalization of $\psi$ and provides a general unitary transformation for it. In the mapped Schrödinger equation it generates a mixed term of the transformed differentials (hidden in the symbol $\mathcal{V}$ in eq. (32)) proportional to the momentum operator $\frac{\hbar}{i} \frac{\partial}{\partial \xi}$

$$
\left(\frac{\hbar}{M} \frac{\partial \epsilon(\xi, \tau)}{\partial \xi}-\frac{1}{A(\tau)} \frac{\partial[\xi A(\tau)+B(\tau)]}{\partial \tau}\right) \frac{\hbar}{i} \frac{\partial \psi(\xi, \tau)}{\partial \xi}
$$

Such a term has no place in Schrödinger's equation, since we do not want to model fields coupling to the particle's momentum. The function in round brackets in eq. (37) has to be zero; its integration with respect to $\xi$ yields

$$
\epsilon(\xi, \tau)=\frac{M}{\hbar A(\tau)} \frac{\partial}{\partial \tau}\left[\frac{\xi^{2}}{2} A(\tau)+\xi B(\tau)+\Xi_{0}(\tau)\right]
$$

Having rid ourselves of the momentum term (37) by insertion of (38) into (36) we turn to the remainder $\mathcal{V}$ in eq. $(32)$. It now represents the time-dependent quadratic potential

$$
\begin{aligned}
\mathcal{V}(\tau)= & \frac{\xi^{2}}{2} \frac{M}{A(\tau)^{2}}\left[A(\tau)\left(\frac{\mathrm{d}^{2}}{\mathrm{~d} \tau^{2}} A(\tau)\right)-2\left(\frac{\mathrm{d}}{\mathrm{d} \tau} A(\tau)\right)^{2}\right]+\xi M\left[\frac{\frac{\mathrm{d}^{2}}{\mathrm{~d} \tau^{2}} B(\tau)}{A(\tau)}-2 \frac{\left(\frac{\mathrm{d}}{\mathrm{d} \tau} B(\tau)\right)\left(\frac{\mathrm{d}}{\mathrm{d} \tau} A(\tau)\right)}{A(\tau)^{2}}\right] \\
& +M\left[\frac{\mathrm{d}}{\mathrm{d} \tau} \Xi_{0}(\tau)-\frac{\left(\frac{\mathrm{d}}{\mathrm{d} \tau} B(\tau)\right)^{2}}{2 A(\tau)^{2}}\right] .
\end{aligned}
$$

Evidently only quadratic potentials with time-dependent frequency [6] can be generated with our approach, the method cannot be generalized to anharmonic potentials!

Through changes of $A, B$ and $\Xi_{0}$ some freedom of choice is available for time-dependent quadratic potentials. For time-dependent quadratic potentials Lewis established a constant of motion in 1967 [7,19], Ray applied it in 1982 [20], as did Agarwal [21] and many others. A recent overview is given in Lohe's 2009 work [22].

To recap our previous examples: eq. (27) is reproduced by $\Xi_{0}(\tau)=\frac{1}{2} \int \mathrm{d} \tau(\partial B(\tau) / \partial \tau)^{2}$ with $A=1$, and $B(\tau)=\frac{a}{2} \tau^{2}$. $\Xi_{0}(\tau)=0, A(\tau)=\exp (i \omega \tau)$ and $B=0$ yields the Schrödinger equation of the harmonic oscillator (10), whereas its "complexification" $A(\tau)=\exp (\omega \tau)$ results in the Schrödinger equation for the inverse oscillator.

\section{Time-dependent harmonic potentials}

We now turn to the general expression for the time-dependent harmonic potentials, those that conform with the form of $\mathcal{V}(\tau)$ found in eq. (39). The reference level of the energy can be chosen in any way we like because the function $\Xi_{0}(\tau)$ gives us complete freedom of choice. We will not investigate the term linear in $\xi$, we choose $B(\tau)=0$. The harmonic term in $\mathcal{V}$ has to conform with the non-linear ordinary differential expression of $A(\tau)$ that forms its coefficient function. This form was previously derived in 1996 by Bluman and Shtelen who argue that it is "arbitrary" [23]. Unfortunately, this does not imply, that it can be determined freely — as one pleases, see Mostafazadeh [24]. Substituting $A(\tau)=\exp [\alpha(\tau)]$ the quadratic term of $\mathcal{V}$ takes the alternative form

$$
\mathcal{V}_{Q}(\tau)=\frac{\xi^{2} M}{2}\left[\frac{\mathrm{d}^{2} \alpha}{\mathrm{d} \tau^{2}}-\left(\frac{\mathrm{d} \alpha}{\mathrm{d} \tau}\right)^{2}\right] .
$$

Here, our main interest is in the application to instantaneous switching of the potential; at the transition time $\tau=\tau_{0}$. In this case, for either version of the form factor (36), be it expressed in terms of $\alpha$ or $A$, we find that the condition for smooth coordinate linkage (15) requires that

$$
A\left(\tau_{0}\right)=1 \quad \text { or } \quad \alpha\left(\tau_{0}\right)=0 .
$$

Two examples illustrate the findings of this section. Firstly, for fig. 4, we choose

$$
A_{1}(\tau)=\exp \left[\frac{1}{8} \tau^{4}+\frac{1}{4} \tau^{2}\right]
$$

with switchover time $\tau_{0}=0$ this conforms with (41). It yields a complicated expression for the time-dependence of the harmonic potential with a simple graphical representation, a positive double-hump that drops sharply to negative values for large values of time, compare inset of fig. 4. 


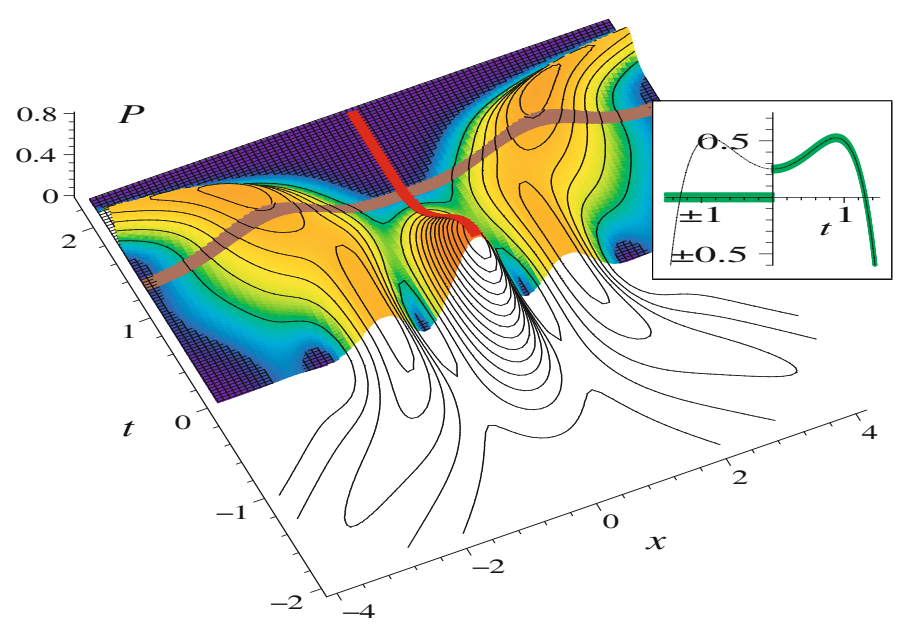

Fig. 4. The time-dependence of the potential's quadratic term $\mathcal{V}_{2} / \xi^{2}$, which can be derived from $A_{1}$ of eq. (42), is shown as a thin line in the inset. The thick green line in the inset describes a scenario where the potential is switched on at the transition time $\tau_{0}=0$, as is shown in the main figure. The free particle's wave function arrives centered with the minimum of the potential (thick red line) in a superposition just like in fig. 1 (here $p_{0}=2$ ). The time dependence of the potential gives rise to focusing of the wave function until, at time $\tau=1.35$ (thick brown line), the potential becomes repulsive.

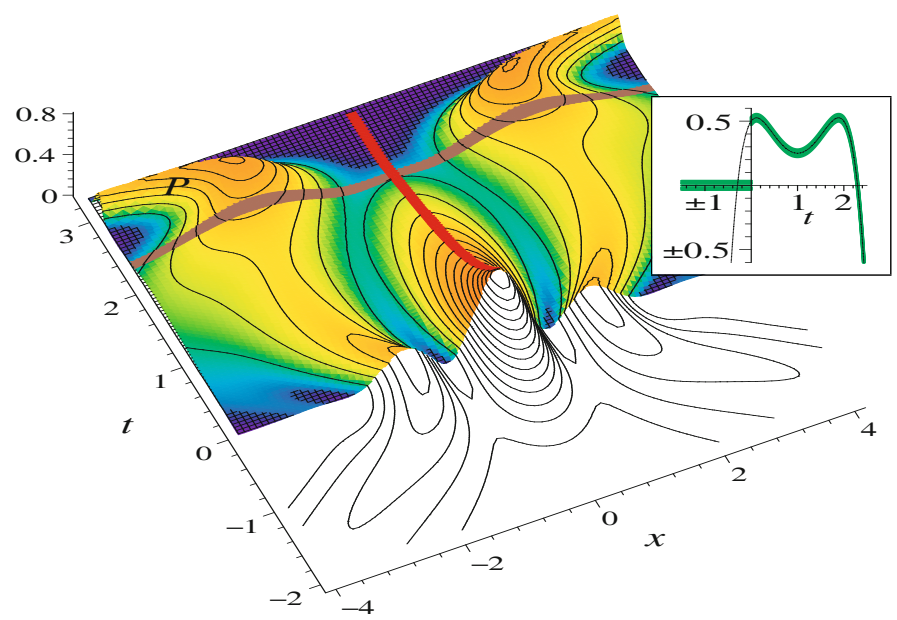

Fig. 5. Essentially the same as fig. 4 , here the time-dependence of the potential's quadratic term $\mathcal{V}_{2} / \xi^{2}$ is derived from $A_{2}$ of eq. (43). After the potential is switched on at $\tau_{0}=0$ it is attractive until at time $\tau=2.3$ (thick brown line) it becomes repulsive.

Secondly, we shift $A_{1}\left(\tau-\frac{1}{2}\right)$ and divide by $A(0)$, to conform with (41), the resulting expression,

$$
A_{2}(\tau)=\exp \left[\frac{1}{8}(\tau-1)^{4}+\frac{1}{4}(\tau-1)^{2}-\frac{3}{8}\right],
$$

was employed to generate fig. 5 .

Note that $\mathcal{V}_{Q}$ is non-linear in $A$ or $\alpha$ which makes even changing the strength of the potential difficult to achieve. Unfortunately, at this stage it appears unlikely that one should be able to succeed in tailoring the time dependence of the harmonic potential to one's needs exactly and still be able to determine $A$ or $\alpha$, by some kind of inversion of eq. (40). Instead, one is therefore forced to use eqs. (39) or (40) to determine $\mathcal{V}$ from $A$ or $\alpha$. At least inversion of $A$ or $\alpha$ is not needed since all relevant expressions (34), (35) and (38) are given in terms of $A$ or $\alpha, B$ and $\Xi_{0}$.

It is, of course, possible to glue together several (or many) slices of maps that approximate a scenario one wants to model.

\section{Conclusions}

General solutions of the free-particle Schrödinger equation can be mapped onto solutions of the Schrödinger equation for the harmonic oscillator using a simple coordinate transformation (7) in conjunction with a multiplication of the 
wave function by a suitable form factor (9). This map is invertible and a composition of two such maps allows us to map from one harmonic oscillator to another with a different, positive or negative, spring constant and different center position. The simplicity of the approach described here makes it a tool of choice for the description of the wave function of a particle experiencing instantaneous transitions from a free to a harmonically trapped state, the instantaneous release from a harmonic trap [3] or the instantaneous change of the stiffness and center position of its harmonic trapping potential.

Instantaneous transitions for particles that are free to ones that are trapped, or vice versa, can experimentally be implemented in quantum optics using optical forces exerted on cold atoms for trapping [25,26], movement [27,28], release and dispersal (using blue-detuned, repulsive trapping fields [26]). Similarly, molecules [29,30] and much larger micro-mechanical quantum systems $[31,32]$ can be manipulated using optical forces. In all these cases the extent of the light field tends to be much greater than the size of the manipulated quantum particle and, since light travels fast, changes to the light field affect the particle's global environment immediately. Switching the light on or off, moving the center of an optical trap or changing the frequency or intensity of the used light is frequently well described by trapping potentials whose spring constant or center position are modified instantaneously "everywhere". All such transitions are easily described with the theory presented here.

The mapping introduced here is computationally more efficient than state-projection or harmonic oscillator propagator techniques and conceptually simpler than mapping techniques such as those used for supersymmetric potentials $[16,17]$. It helps with symbolic calculations because it allows for the determination of the time evolution of wave functions trapped in a harmonic potential using the lower computational overheads of wave functions evolving in free space [3].

For numerical calculations the trapped case provides better control than the free case since the particle is spatially confined. One can determine the largest momentum components and thus work out the maximal harmonic oscillation amplitude and smallest expected feature size in space. In time, on account of the harmonic oscillator's periodicity, at most half an oscillation period is needed. Accordingly, a suitable static grid for numerical calculations is easily devised.

I would like to thank Georg Ritter, Dimitris Kakofengitis, and Gary Bowman for valuable feedback on the manuscript.

Note added in proofs.

I am grateful to Julio Guerrero for pointing me to refs. [33-35] introducing similar concepts as discussed here using abstract conservation laws.

Open Access This is an open access article distributed under the terms of the Creative Commons Attribution License (http://creativecommons.org/licenses/by/4.0), which permits unrestricted use, distribution, and reproduction in any medium, provided the original work is properly cited.

\section{References}

1. O. Steuernagel, Am. J. Phys. 73, 625 (2005) arXiv:physics/0312116v2.

2. O. Steuernagel, D. Kakofengitis, G. Ritter, Phys. Rev. Lett. 110, 030401 (2013) arXiv:1208.2970.

3. O. Steuernagel, arXiv:1109.1818 (2011).

4. A. Yariv, Quantum Electronics (Wiley, New York, 1967).

5. U. Niederer, Helv. Phys. Acta 45, 802 (1972).

6. S. Takagi, Prog. Theor. Phys. 84, 1019 (1990).

7. H.R. Lewis, Phys. Rev. Lett. 18, 510 (1967).

8. G. Nienhuis, L. Allen, Phys. Rev. A 48, 656 (1993).

9. G. Barton, Ann. Phys. 166, 322 (1986).

10. C. Yuce, A. Kilic, A. Coruh, Phys. Scr. 74, 114 (2006) arXiv:quant-ph/0703234.

11. K. Andrzejewski, J. Gonera, P. Kosinski, arXiv:1310.2799 (2013).

12. T. Kiss, J. Janszky, P. Adam, Phys. Rev. A 49, 4935 (1994).

13. H. Moya-Cessa, M.F. Guasti, Phys. Lett. A 311, 1 (2003).

14. H. Goldstein, Classical Mechanics (Addison-Wesley, Reading, 1981).

15. W.P. Schleich, Quantum Optics in Phase Space (Wiley-VCH, Berlin, 2001).

16. L.É. Gendenshteïn, Sov. J. Exp. Theor. Phys. Lett. 38, 356 (1983).

17. F. Cooper, J.N. Ginocchio, A. Wipf, J. Phys. A: Math. Gen. 22, 3707 (1989).

18. R.N.C. Filho, G. Alencar, B.S. Skagerstam, J.S. Andrade, EPL 101, 10009 (2013).

19. H.R. Lewis, Jr., J. Math. Phys. 9, 1976 (1968).

20. J.R. Ray, Phys. Rev. A 26, 729 (1982).

21. G.S. Agarwal, S.A. Kumar, Phys. Rev. Lett. 67, 3665 (1991).

22. M.A. Lohe, J. Phys. A: Math. Theor. 42, 035307 (2009). 
23. G. Bluman, V. Shtelen, J. Phys. A: Math. Gen. 29, 4473 (1996).

24. A. Mostafazadeh, J. Math. Phys. 40, 3311 (1999).

25. S. Chu, Rev. Mod. Phys. 70, 685 (1998).

26. R. Grimm, M. Weidemüller, Y.B. Ovchinnikov, arXiv:physics/9902072 (1999).

27. A. Walther, F. Ziesel, T. Ruster, S.T. Dawkins, K. Ott, M. Hettrich, K. Singer, F. Schmidt-Kaler, U. Poschinger, Phys. Rev. Lett. 109, 080501 (2012).

28. R. Bowler, J. Gaebler, Y. Lin, T.R. Tan, D. Hanneke, J.D. Jost, J.P. Home, D. Leibfried, D.J. Wineland, Phys. Rev. Lett. 109, $080502(2012)$.

29. E.S. Shuman, J.F. Barry, D. Demille, Nature 467, 820 (2010) arXiv:1103.6004.

30. I. Manai, R. Horchani, H. Lignier, P. Pillet, D. Comparat, A. Fioretti, M. Allegrini, Phys. Rev. Lett. 109, 183001 (2012) arXiv: 1211.2652

31. T.J. Kippenberg, K.J. Vahala, Science 321, 1172 (2008).

32. F. Marquardt, S.M. Girvin, Physics 2, 40 (2009).

33. M. Fernández Guasti, H. Moya-Cessa, Phys. Rev. A 67, 063803 (2003) quant-ph/0212073.

34. J.F. Cariñena, J. de Lucas, M.F. Rañada, SIGMA 4, 031 (2008) math-ph/0803.1824.

35. J. Guerrero, F.F. López-Ruiz, V. Aldaya, F. Cossío, J. Phys. A Math. Gen. 44, 44537 (2011) quant-ph/1010.5525. 